$1-1-2021$

\title{
Factors influencing HIV care outcomes among adolescents living with HIV in rural South Africa
}

Lindsey M. Filiatreau

Audrey Pettifor

Jess Edwards

Nkosinathi Masilela

Rhian Twine

See next page for additional authors

Follow this and additional works at: https://knowledgecommons.popcouncil.org/departments_sbsr-hiv

Part of the Public Health Commons

How does access to this work benefit you? Let us know!

\section{Recommended Citation}

Filiatreau, Lindsey M., Audrey Pettifor, Jess Edwards, Nkosinathi Masilela, Rhian Twine, F. Xavier GómezOlivé, Nicole Haberland, Chodziwadziwa Whiteson Kabudula, Sheri A. Lippman, and Kathleen Kahn. 2021. "Factors influencing HIV care outcomes among adolescents living with HIV in rural South Africa," Project SOAR Results Brief. Washington, DC: Population Council. 


\section{Authors}

Lindsey M. Filiatreau, Audrey Pettifor, Jess Edwards, Nkosinathi Masilela, Rhian Twine, F. Xavier GómezOlivé, Nicole Haberland, Chodziwadziwa Whiteson Kabudula, Sheri A. Lippman, and Kathleen Kahn 


\section{Factors Influencing HIV Care Outcomes among Adolescents Living With HIV in Rural South Africa}

\section{EXECUTIVE SUMMARY}

\section{Methods}

- Cross-sectional survey conducted among adolescents and young people ages 12-24 living with HIV in the Agincourt Health and Socio-Demographic Surveillance System site in rural Mpumalanga Province, South Africa.

- Recruitment by study staff, healthcare providers, and area home-based care workers using Tier.net, South Africa's national HIV care outcome database, and Clinic Link, an extant, population-based clinical care database.

- Measures of interest: disclosure, HIV-related stigma, depression, social support, resilience, self-esteem, perceived stress, history of physical or sexual violence, substance use, and self-reported HIV care and care-seeking outcomes.

\section{Key findings}

- Overall, self-reported engagement in HIV care is high in this sample of young people ages 12-24. Just over 85 percent of individuals reported no missed ART doses in the prior 30 days.

- Psychosocial factors significantly affect young people's HIV care outcomes:

- Among adolescent boys and young men (ABYM) living with HIV, older age and prior history of sexual violence are significantly associated with treatment non-adherence/ loss to care. Higher levels of social support are protective/significantly associated with adherence.

- Among adolescent girls and young women (AGYW), higher levels of depression and of perceived stress are significantly associated with treatment non-adherence/ loss to care.

- Prevalence of psychosocial challenges increase with age: Individuals ages 18-24 that are living with HIV report higher levels of perceived stress and depression and lower levels of social support than those under the age of 18.

- Contrary to our expectations, stigma, substance use, physical partner violence, and physical family violence were not associated with treatment adherence for males or females.

\section{Key conclusions}

- Interventions aimed at improving emotional well-being and social support among adolescents and young adults living with HIV are crucial to improving HIV care outcomes within this highly vulnerable population.

- Differentiated care models that are specifically designed to address both the clinical and psychosocial needs of young people could yield improvements in retention in care and ART adherence among young people living with HIV. 


\section{BACKGROUND}

Adolescents continue to bear a disproportionate burden of the HIV epidemic worldwide. In South Africa specifically, HIV remains the second leading natural cause of death among young people ages 15-24. ${ }^{1}$ Access to antiretroviral therapy (ART) has gradually expanded in the country over the past decade culminating in the adoption of the World Health Organization's Universal Test and Treat (UTT) policy in September 2016. This policy expanded access to ART to all South Africans living with HIV for the first time ${ }^{2,3}$ and has yielded substantial improvements in the proportion of people living with HIV (PLHIV) that are in care and virally suppressed. ${ }^{4}$ However, disparities in care outcomes between adolescents and adults living with HIV persist. ${ }^{5}$ The most recent country estimates suggest that while 63 percent of PLHIV ages 25-49 are currently on ART, just 40 percent of HIV-positive young people ages 15-24 are taking treatment. ${ }^{6,7}$

Adolescence is a time of physical and mental maturation, as well as identity experimentation. ${ }^{8}$ It is a period characterized by poor mental health outcomes, limited social support, increased vulnerability to stigma and violence, and a lack of access to age-appropriate healthcare services. ${ }^{9-15}$ Prior research suggests the aforementioned factors could contribute to poor HIV care outcomes among adolescents, ${ }^{9,10,14-21}$ which can be further exacerbated in rural contexts where strains on the healthcare system often limit care differentiation and adequate service delivery. ${ }^{22,23}$ If South Africa is to meet the Joint United Nations Program on HIV and AIDS 90-90-90 targets, it is essential to identify and address factors driving negative HIV care outcomes among HIV-positive young people in rural areas in the era of UTT. ${ }^{9,24,25}$

In the Agincourt Health and Socio-Demographic Surveillance System (HDSS) site in rural Mpumalanga Province, we hypothesized that HIV status disclosure, individuals' prior healthcare seeking experiences, history of depression and overall emotional well-being, experiences with domestic and intimate partner violence, and substance use could contribute to poor levels of treatment adherence among adolescents living with
HIV. The overall objective of this study was to better understand HIV care outcomes among HIV-positive adolescents in the Agincourt HDSS in the era of UTT so we can better inform HIV care programs targeting this vulnerable population.

\section{METHODS}

We conducted a cross-sectional survey among HIV-positive young people ages 12-24 living and accessing care within the HDSS site. HIV-positive young people were recruited for study participation using household location data from Tier.net, South Africa's national HIV treatment and care database, and Clinic Link, an extant database that links consenting HDSS residents' clinical records to their annual census data. Recruitment was conducted by study staff, healthcare facility employees, and area home-based care workers.

Locally hired, trained research assistants fluent in Xitsonga, the local language, administered the study questionnaire to consenting/assenting participants recruited from their households using data from the previously described datasets. The questionnaire captured data on participants' demographics, experiences living with HIV (prior history of disclosure, HIV-related stigma [Berger HIV Stigma Scale $\left.{ }^{26}\right]$ ), emotional well-being (depression-20-item CES-D;27-29 social support-modified 8-item Medical Outcomes Social Support Survey; ${ }^{30}$ self-esteem-10item Rosenburg Self-Esteem Scale, ${ }^{31}$ resilience25-item Conner Davidson Resilience scale; ${ }^{32,33}$ perceived stress-10-item Sheldon Cohen Perceived Stress Scale ${ }^{34}$ ), history of violence (physical or sexual violence-instrument developed by the World Health Organization ${ }^{35}$ ), substance use (Alcohol, Smoking, and Substance Involvement Screening Test), prior healthcare seeking experiences within the HDSS, and self-reported HIV care outcomes.

Descriptive statistics ( $\mathrm{n} /$ proportions or medians/ interquartile ranges) were calculated to characterize the study population overall, and by self-reported adherence to ART. Overall HIV care outcomes were summarized using an HIV care cascade framework, for which we estimated the proportion of study participants that had ever sought HIV-related care, 
the proportion of those individuals that had ever initiated ART, the proportion of those that were currently on ART, and the proportion of those that reported no missed ART doses within the prior 30 days. Log binomial regression was used to assess the association between exposures of interest and the primary composite outcome of: 1) being presently out of HIV care or 2) missing more than seven doses of prescribed ART at any point in the prior 12 months. All analyses were stratified by participant gender and were conducted using SAS Studio (Cary, NC).

\section{RESULTS}

A total of 362 young people ages 12 to 24 (median=21 years) were included in this study. Just over 70 percent of the study population was 18 or older ( $n=256)$, most were female $(n=254,70 \%)$, unemployed $(n=168,46 \%)$, and single $(n=297$, $82 \%)$. Over half of participants were single or double orphans $(n=190)$, and over 75 percent of participants $(n=277 ; 77 \%)$ had completed at least some secondary school (Table 1).

When compared to female participants, male participants were substantially younger ( $48 \%$ of male participants were under the age of 18 versus $21 \%$ of female participants). Male participants' younger age may partly explain why they were less likely to be in a relationship than female participants (5\% versus $37 \%)$, were more likely to be current students (69\% versus 38\%), and reported lower education levels ( $19 \%$ of male participants had completed secondary school at the time of the survey versus $36 \%$ of female participants).

\section{Self-reported exposures of interest}

\section{HIV-related measures}

- Disclosure: Just over 54 percent ( $n=179)$ of study participants reported they had previously disclosed their HIV status to at least one other person

- Disclosure varied by gender, with ABYM less likely to report disclosure than AGYW (31\% versus $64 \%$; $p<0.0001$ ).
- Disclosure varied by age: Approximately 70 percent of young people ages 18-24 reported prior disclosure of their status, while just 20 percent of respondents under the age of 18 had disclosed $(p<0.0001)$. The two most commonly reported reasons for choosing not to disclose among individuals under the age of 18 were because they did not know enough about HIV and because they were concerned the individual they wanted to tell might tell others.

- Of the 43 participants who reported being married or in a relationship and responded to the question about partner disclosure (20 individuals did not answer), most ( $n=26 ; 61 \%$ ) individuals reported they had not disclosed to their partner. Of the 41 married/partnered AGYW who responded to the question, only a third $(n=15)$ reported they had disclosed to their partner. There were too few ABYM ( $n=5$ reported being in a relationship) to observe patterns.

- Stigma: Median score on the Berger HIV Stigma scale, which captures respondents' personalized stigma, disclosure concerns, negative self-image, and perceptions of public attitudes, was 24 (IQR: 21-27). Possible scale scores range from 10 to 40 , indicating study respondents had slightly lower perceived stigma than the median possible scale score of 25. Stigma scores did not substantially vary by age or gender (Table 1 ).

\section{Emotional well-being}

- Overall, for indicators of emotional well-being, there were no differences between males and females, however there were differences between older and younger respondents

- Depression: Over one quarter of participants ( $n=102 ; 27 \%)$ had results indicative of depression at a CES-D cutoff of 16. Possible CES-D scores range from 0 to 60 , with 16 routinely used as a cut-point for depression. Depression was higher in individuals ages 18-24, with 31 percent of young people 18-24 scoring 16 or higher on the CES-D, compared to $19 \%$ among individuals under the age of $18(p=0.012)$. 
Table 1 Respondent characteristics

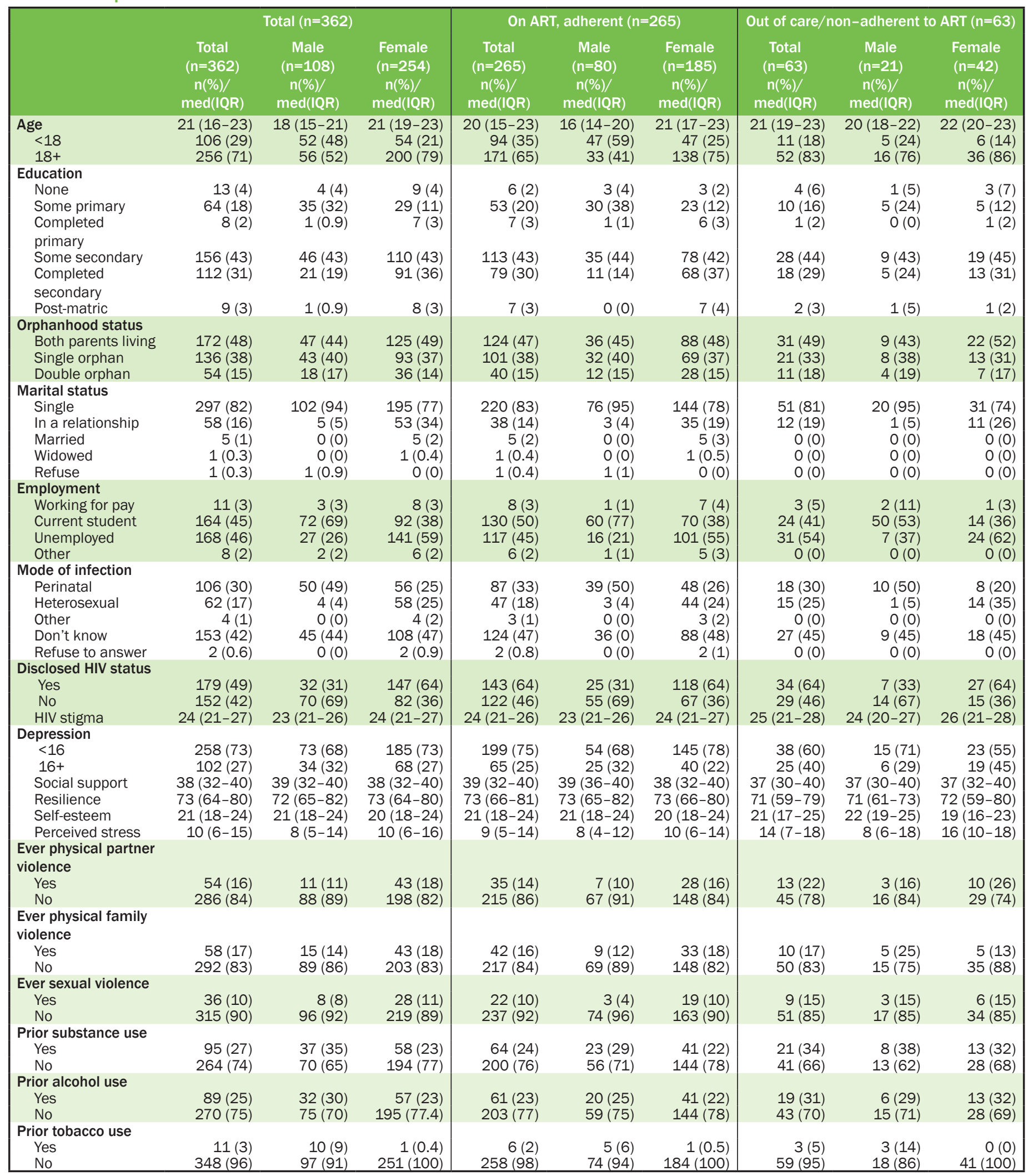

*Missing: Adherence status-34; Employment-11; Mode of infection-35; Disclosure-31; Stigma-36; Depression-2; Social support-9; Resilience-16; Self esteem-11; Perceived stress-7; Physical partner violence-22; Physical family violence-12; Sexual violence-11; Substance use-3; Alcohol use-3; Tobacco use-3. 
- Social support: Reported social support was high among study respondents with a median overall score of 38 , just two points lower than the maximum possible score (possible scale scores range from 8 to 40). Young adults had lower reported social support (mean: 34.3 ) when compared to adolescents under the age of 18 (mean: 37.5$)(p<0.0001)$.

- Resilience: Overall resilience was high in comparison to other studies among adolescents in South Africa, with a median score of 73 (IQR: 64-80). ${ }^{36,37}$

- Perceived stress: Perceived stress within the population was low in comparison to other populations within South Africa. ${ }^{38}$ Perceived stress was higher among young people ages 18-24 (median: 12; IQR: 7-16) when compared to individuals under the age of 18 (median: 6; IQR: $4-10)(p<0.0001)$.

\section{Violence}

- Overall, individuals over the age of 18 were more likely to report prior history of physical violence perpetuated by a partner $(p=0.002)$ or family member $(p=0.026)$, as well as sexual violence $(p=0.032)$. No significant differences were observed in reported violence by gender.

- Physical intimate partner violence: Prior experiences of intimate partner violence was reported by approximately 25 percent of participants, with 27 percent of female respondents reporting history of IPV and 17 percent of male respondents.

- Physical family violence: Prior experiences of violence perpetuated by a family member or friend was lower than partner violence, with just 17 percent of respondents reporting a history of physical violence perpetuated by a family member or friend. Proportions of individuals experiencing family violence were similar between men and women.

- Sexual violence: Thirty-six of 351 individuals (10\%) reported they had been forced to have sexual intercourse when they did not want to or had sexual intercourse in the past because they were afraid of what the individual might do. While the prevalence of sexual violence/coercion was higher among females than males (11\% vs. $8 \%$ ), this was not significantly different, possibly due to the small sample.

\section{Substance use}

- Overall substance use within the population was lower than expected, with just two individuals reporting use of substances other than tobacco or alcohol within their lifetime. One individual reported prior use of inhalants (no use within prior three months) and one individual reported prior use of cannabis (no use within prior three months). Including use of alcohol and tobacco, just 27 percent of respondents reported prior substance use within their lifetime.

- Alcohol use: Among the 89 individuals who reported prior history of alcohol use, just 11 individuals, eight men and three women, reported daily or weekly use in the past three months.

- Tobacco use: Reported tobacco use was very low within the population with just $11(3 \%)$ individuals reporting prior use, 10 males and one female. Of these 11 individuals, 8 (73\%) reported daily or weekly tobacco use within the prior three months.

\section{Prior healthcare-seeking experiences in the HDSS}

- Overall, participants reported positive prior healthcare seeking experiences within the HDSS. Just 7 percent of individuals reported difficulty accessing care within the past year, and just 7 percent of individuals would not recommend the last clinic they attended to a friend. ABYM reported difficulty accessing care more frequently than AGYW, though differences were not statistically significant ( $9 \%$ versus $6 \%$; $p=0.22$ ).

- Clinic bypassing: Approximately 15 percent $(n=56)$ of study participants reported bypassing the closest clinic to their home, or electing to travel farther from home for preferred care, the last time they sought care. There were no significant differences observed by gender (16\% in females versus $14 \%$ in males; $p=0.59)$ or age $(13 \%$ of 
individuals under age 18 versus $16 \%$ of individuals ages $18+; p=0.44)$.

\section{Self-reported HIV care outcomes of interest}

- Thirty-one individuals ( $9 \%$ of the study population) stated they were HIV-negative when asked about their HIV care-seeking experiences, despite the fact that participants were contacted based on a positive HIV test result documented in their clinical records. This could point to denial about serostatus, or discomfort in speaking to study staff about their status.

- Mode of infection: Of 331 respondents, just over 50 percent $(n=174)$ of young people overall reported knowledge of how they became infected with HIV. Substantially fewer young people ages 18-24 reported knowledge of how they became infected, with 49 percent stating they were unsure of infectious route (versus 39\% of adolescents under the age of 18). This may indicate that older individuals, who are more likely to be infected through heterosexual transmission, are less likely to disclose infection via sexual transmission. Male participants were less likely to report heterosexual contact as the mode of infection (4\% versus 25\% of female participants) than females, with most ABYM reporting they either did not know how they became infected $(n=45 ; 44 \%)$, or were infected through perinatal transmission $(n=50 ; 49 \%)$.
- Knowledge of viral load/CD4 cell counts: Two hundred fifty-eight individuals (of 265 respondents; 97\%) reported they did not know their most recent viral load result when asked. ${ }^{\text {a }}$ Two hundred fifteen individuals (of 229 respondents; 94\%) stated they did not know their most recent CD4 test result. ${ }^{b}$

- ABYM, though more likely to self-report seeking care, starting ART, and current ART use, were less likely to report a high level of adherence in the prior 30 days than young women, with 77 percent of men who were currently on ART reporting no missed ART doses in the prior 30 days, versus 89 percent of young women (Figure 1).

- Individuals under the age of 18 experienced better care outcomes across the continuum than young adults (Figure 1).

\section{Associations with treatment non- adherence}

Unadjusted regression analyses suggest age, depression, social support, resilience, and perceived stress are associated with the composite outcome of being non-adherent to ART or out of care in the overall study sample (Table 2). When stratifying by

\footnotetext{
Two additional individuals reported implausible results-a
} viral load of 1 and 2

${ }^{\text {b} T h r e e ~ a d d i t i o n a l ~ p a r t i c i p a n t s ~ r e p o r t e d ~ t h e ~ y e a r ~ o f ~ t e s t i n g ~ a s ~}$ opposed to the lab result value (2018 and 2019).

\section{Figure 1 HIV care outcomes by populations of interest}

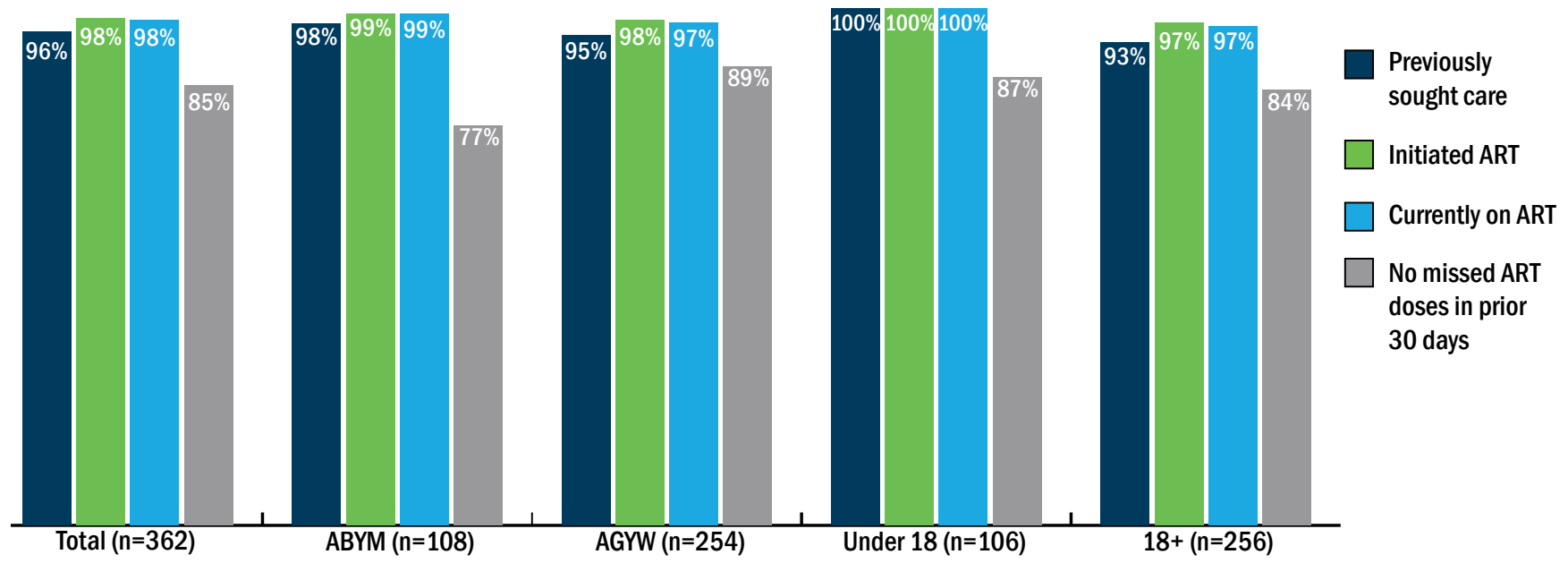

*Reported percentages are based off total $\mathrm{N}$ from preceding stage in care continuum 
Table 2 Unadjusted prevalence ratio estimates of treatment non-adherence

\begin{tabular}{|llll|}
\hline & Total & ABYM & AGYW \\
UgPR $(95 \%$ Cl $)$
\end{tabular}

*indicates significance at $p<0.05$

gender, higher levels of depression and perceived stress were significantly associated with nonadherence or loss to care among AGYW, while older age, history of sexual violence, and lower levels of social support were significantly associated with nonadherence or loss to care among ABYM (Table 2).

\section{DISCUSSION}

The proportion of study participants reporting prior care-seeking for HIV-related services, a history of treatment use, and current treatment use was high when compared to national estimates among adolescents living with HIV. ${ }^{6}$ About 85 percent of the sample self-reported adherence to ART in the prior 30 days. Young people ages 18-24 experienced poorer care outcomes across the continuum than adolescents under the age of 18 , potentially due to the fact that young people ages 18-24 also reported poorer emotional well-being and less social support than adolescents. We did not assess services provided specifically to younger adolescents within the study site, but we believe the differences in emotional well-being outcomes between adolescents and young adults are likely attributable to factors such as vertical versus horizontal infection and social protection obtained through primary and secondary school attendance. The proportion of young people and adolescents who reported prior care-seeking, ART initiation, and current ART use could also be inflated because of social desirability bias among survey respondents, or issues with generalizability, as individuals in care may have been easier to locate and more willing to participate in the study than individuals that had fallen out of care. Corroborating findings using clinical record data, which is currently underway, could increase confidence in the reliability of study findings.

Emotional well-being and social support were significantly associated with retention in care and ART adherence among young people living with HIV overall, though differences were observed in these results when stratifying by gender. In the study population, poorer social support, higher perceived stress, and indications of depression were significantly associated with being out of care or nonadherent to ART. Among AGYW, ART non-adherence was specifically associated with depression and higher levels of perceived stress. Among ABYM, ART non-adherence was specifically associated with lower levels of social support. Given that participants over the age of 18 were more often depressed, had less social support, and reported higher levels of 
perceived stress than adolescents 13-17 years, improving emotional well-being and social support could be particularly important in improving care outcomes among young people ages 18-24 living with HIV.

Though a greater proportion of ABYM reported prior care seeking, ART initiation, and current ART use than AGYW, a smaller proportion of ABYM reported being ART adherent in the past 30 days. These differences in care outcomes by gender were statistically insignificant, but trends could be attributable to the fact that men also reported greater difficulty getting the care they needed in the prior year than women. Additionally, just 31 percent of young men reported prior disclosure of their HIV status. Given that disclosure is known to improve ART adherence among people living with HIV, ${ }^{39-41}$ helping young men expand their social support system, identify a trusted-individual within that support system to whom they could disclose their HIV status, and disclose their status to that trusted individual has the potential to improve ART adherence among young men living with HIV.

Results from this study also suggest that prior history of sexual violence significantly influences ART adherence among ABYM. Within South Africa, men are most commonly viewed as perpetrators of violence. However, results from this study suggest that 11 percent of HIV-positive young men are survivors of physical partner violence, 14 percent of physical violence perpetrated by a friend or family member, and 8 percent of sexual violence, with some men reporting multiple types of experienced violence. Non-adherence among young men reporting prior history of sexual violence was 2.68 times higher than among young men with no reported history of sexual violence. Addressing current gaps in support services for male survivors of sexual violence could improve ART adherence among young men living with HIV. A large proportion of young men in our study population were under the age of 18 and reported infection through vertical transmission. As a result, our results may not be generalizable to men who were sexually infected.

\section{CONCLUSIONS}

Overall emotional well-being and social support is significantly associated with ART adherence among young people. Among AGYW, history of depression and overall perceived stress significantly influence ART adherence. Among ABYM, social support and prior history of sexual violence significantly influence ART adherence.

Mental health care is lacking, particularly in rural areas. Treatment for depression is not available in most public sector clinics, nor is access to mental health professionals. Individuals seeking mental healthcare or showing signs of severe depression may be referred to an overstretched social work system. Interventions that can be delivered at the clinical level are necessary for improving overall emotional well-being and ART adherence among young people, specifically young women, living with HIV within this context.

Social support is also key in improving retention in care and ART adherence among HIV-positive young people, particularly young men. Empirical evidence demonstrates the importance of treatment support and peer group support in improving care outcomes for youth, yet is lacking from many HIV care facilities. There should be a greater investment in scaling up evidence-based programs for adolescents such as the Zvandiri program, ${ }^{42}$ that provide comprehensive, differentiated prevention, treatment, and care specific to the needs of this highly vulnerable population, a crucial component in improving HIV care outcomes across the continuum.

\section{REFERENCES}

1. Statistics South Africa. 2017. "Mortality and causes of death in South Africa, 2015: Findings from death notification."

2. WHO. 2015. Guideline on When to Start Antiretroviral Therapy and on Pre-exposure Prophylaxis for HIV. Geneva: WHO.

3. Pillay, Y. \& A. Pillay. 2016. Implementation of the Universal Test and Treat strategy for HIV positive patients and differentiated care for stable patients. 
4. Lippman, S. A. et al. 2019. "Improvements in the South African HIV care cascade: findings on 90-90-90 targets from successive population-representative surveys in North West Province," Journal of the International AIDS Society 22(6): e25295. doi: 10.1002/jia2.25295

5. Enane, L. A. et al. 2018. "Traversing the cascade: urgent research priorities for implementing the 'treat all' strategy for children and adolescents living with HIV in sub-Saharan Africa," Journal of Virus Eradication 4(Suppl 2): 40-46.

6. Simbayi, L. C., et al. 2019. South African National HIV Prevalence, Incidence, Behaviour and Communication Survey, 2017. Cape Town: HSRC Press.

7. Human Sciences Research Council (HSRC). 2018. "The Fifth South Africa national HIV prevalence, incidence, behavioral and communication survey, 2017 overview presentation." doi:10.15713/ins.mmj.3

8. Millstein, S., A. Petersen and E. Nightingale. 1994. Promoting the Health of Adolescents: New directions for the twenty-first century. New York: Oxford University Press Inc. doi:10.1097/00006205-199402000-00023

9. Davies, M. A. and J. Pinto. 2015. "Targeting 90-90-90Don't leave children and adolescents behind," Journal of the International AIDS Society 18(7Suppl 6): 20745. doi: 10.7448/IAS.18.7.20745

10. Fortenberry, J. D. et al. 2012. "Linkage to care for HIVpositive adolescents: A multisite study of the adolescent medicine trials units of the adolescent trials network," Journal of Adolescent Health 51(6): 551-6. doi: 10.1016/j.jadohealth.2012.03.012.

11. Acree, M. E. 2017. "Transition of care for youth with HIV," Pediatric Annals 46(5): e198-e202. doi: 10.3928/19382359-20170424-02

12. Murphy, D. A. et al. 2005. "Longitudinal antiretroviral adherence among adolescents infected with human immunodeficiency virus," Archives of Pediatrics \& Adolescent Medicine 159(8):764-70. doi: 10.1001/ archpedi.159.8.764

13. Naswa, S. \& Y. S. Marfatia. 2010. "Adolescent HIV/ AIDS: Issues and challenges," Indian Journal of Sexually Transmitted Diseases 31(10): 1-10. doi: 10.4103/25890557.68993

14. Lee, S. J. et al. 2007. "The effect of social support on mental and behavioral outcomes among adolescents with parents with HIV/AIDS," American Journal of Public Health 97(10): 1820-6. doi: 10.2105/ AJPH.2005.084871

15. Cluver, L. et al. 2018. "STACKing the odds for adolescent survival: health service factors associated with full retention in care and adherence amongst adolescents living with HIV in South Africa," Journal of the International AIDS Society 21(9): e25176. doi: 10.1002/ jia2.25176
16. Camara, M., G. Bacigalupe, and P. Padilla. 2017. "The role of social support in adolescents: Are you helping me or stressing me out?," International Journal of Adolescent Youth 22(2): 123-136. doi: 10.1080/02673843.2013.875480

17. Lall, P. et al. 2015. "Review: An urgent need for research on factors impacting adherence to and retention in care among HIV-positive youth and adolescents from key populations," Journal of the International AIDS Society 18(2Suppl 1): 19393. doi: 10.7448/IAS.18.2.19393

18. Pettifor, A. et al. 2018. "Adolescent lives matter: Preventing HIV in adolescents," Current Opinion in HIV and AIDS 13(3): 265-273. doi: 10.1097/ COH.0000000000000453

19. Patel, V. et al. 2007. "Mental health of young people: A global public-health challenge," The Lancet 369(9569): 1302-1313.doi: 10.1016/S0140-6736(07)60368-7

20. UNAIDS. 2018. "Miles to go-Closing gaps, breaking barriers, righting injustices." Geneva: UNAIDS.

21. Mark, D. et al. 2017. "HIV treatment and care services for adolescents: A situational analysis of 218 facilities in 23 sub-Saharan African countries," Journal of the International AIDS Society 20(Suppl 3): 21591. doi: 10.7448/IAS.20.4.21591

22. Booysen, F. le R. 2003. "Urban-rural inequalities in health care delivery in South Africa," Development Southern Africa 20(5): 659-673. doi: 10.1080/0376835032000149298

23. Versteeg, M., L. du Toit, and I. Couper. 2013. “Building consensus on key priorities for rural health care in South Africa using the Delphi technique," Global Health Action 6: 19522. doi: 10.3402/gha.v6i0.19522.

24. WHO. 2013. "HIV and adolescents: Guidance for HIV testing and counseling and care for adolescents living with HIV." Geneva: WHO.

25. Wong, V. J. et al. 2017. "Adolescents, young people, and the 90-90-90 goals: A call to improve HIV testing and linkage to treatment," AIDS 31(Suppl 3): S191-S194. doi: 10.1097/QAD.0000000000001539.

26. Wright, K. et al. 2007. "Stigma scale revised: reliability and validity of a brief measure of stigma for HIV+ youth," Journal of Adolescent Health 40(1): 96-98. doi: 10.1016/j.jadohealth.2006.08.001

27. Siddaway, A. P., A. M. Wood and P. J. Taylor. 2017. "The Center for Epidemiologic Studies-Depression (CES-D) scale measures a continuum from well-being to depression: Testing two key predictions of positive clinical psychology," Journal of Affective Disorders 213: 180-186. doi: 10.1016/j.jad.2017.02.015

28. Phillips, G. A. et al. 2006. “The center for epidemiologic studies depression scale with a young adolescent population: A confirmatory factor analysis," Multivariate Behavioral Research 41(2): 147-63. doi: 10.1207/ s15327906mbr4102_3 
29. Eaton, W. 2004. Center for Epidemiologic Studies Depression Scale: Review and Revision (CESD and CESD-R). In M. E. Maruish (Ed.), The use of psychological testing for treatment planning and outcomes assessment: Instruments for adults (p. 363-377). Lawrence Erlbaum Associates Publishers.

30. Moser, A. et al. 2012. "The eight-item modified Medical Outcomes Study Social Support Survey: Psychometric evaluation showed excellent performance," Journal of Clinical Epidemiology 65(10): 1107-16. doi: 10.1016/j. jclinepi.2012.04.007

31. Rosenberg, M. 1965. Society and the Adolescent SelfImage. Princeton, NJ: Princeton University Press.

32. Jørgensen, I. E. and S. Seedat. 2008. "Factor structure of the Connor-Davidson Resilience Scale in South African adolescents," International Journal of Adolescent Medicine and Health 20(1): 23-32. doi: 10.1515/ IJAMH.2008.20.1.23

33. Connor, K. and J. Davidson. 2003. Connor-Davidson Resilience Scale (CD-RISC). 2, 76-82.

34. Cohen, S. 1994. Perceived Stress Scale.

35. Garcia-Moreno, C. et al. 2005. "WHO multi-country study on women's health and domestic violence against women: Initial results on prevalence, health outcomes and women's responses." Geneva, Switzerland: World Health Organization.

36. Bruwer, B. et al. 2008. "Psychometric properties of the Multidimensional Scale of Perceived Social Support in youth," Comprehensive Psychiatry 49(2): 195-201. doi: 10.1016/j.comppsych.2007.09.002

37. Fincham, D. S. et al. 2009. "Posttraumatic stress disorder symptoms in adolescents: risk factors versus resilience moderation," Comprehensive Psychiatry 50(3): 193-9. doi: 10.1016/j.comppsych.2008.09.001
38. Hamad, R. et al. 2008. "Social and economic correlates of depressive symptoms and perceived stress in South African adults," Journal of Epidemiology and Community Health 62(6): 538-44. doi: 10.1136/jech.2007.066191

39. Evangeli, M. and C. Foster. 2014. "Who, then what? The need for interventions to help young people with perinatally acquired HIV disclose their HIV status to others," AIDS 28(Suppl 3): S343-6. doi: 10.1097/ QAD.0000000000000334.

40. Sithole, Z. et al. 2018. “Virological failure among adolescents on ART, Harare City, 2017-a case-control study," BMC Infectious Diseases 18(1): 469. doi: 10.1186/s12879-018-3372-6

41. Bikaako-Kajura, W. et al. 2006. "Disclosure of HIV status and adherence to daily drug regimens among HIVinfected children in Uganda," AIDS and Behavior 10(4 Suppl): S85-93. doi: 10.1007/s10461-006-9141-3

42. Willis, N. et al. 2018. "Zvandiri-bringing a differentiated service delivery program to scale for children, adolescents, and young people in Zimbabwe," Journal of Acquired Immune Deficiency Syndromes 78(Suppl 2): S115-S123. doi: 10.1097/QAI.0000000000001737

Suggested citation: Filiatreau, Lindsey M., Audrey Pettifor, Jessie K. Edwards, Nkosinathi Masilela, Rhian Twine, F. Xavier Gómez-Olivé, Nicole Haberland, Chodziwadziwa Whiteson Kabudula, Sheri A. Lippman, and Kathleen Kahn. 2021. "Factors influencing HIV care outcomes among adolescents living with HIV in rural South Africa," Project SOAR Results Brief. Washington, DC: Population Council.
Project SOAR is a six-year+ (September 2014-January 2021) cooperative agreement funded by the U. S. President's Emergency Plan for AIDS Relief (PEPFAR) and the U. S. Agency for International Development (Agreement No. AID-OAA-A-14-00060). The contents of this brief are the sole responsibility of Project SOAR and Population Council and do not necessarily reflect the views of PEPFAR, USAID, or the United States Government.

Population Council leads the Project SOAR consortium in collaboration with Avenir Health, Elizabeth Glaser Pediatric AIDS Foundation, the Johns Hopkins University, Palladium, and The University of North Carolina at Chapel Hill.
Project SOAR/Population Council 4301 Connecticut Avenue, NW, Suite 280 Washington, DC 20008

Tel: +1 2022379400

e-mail: ProjectSOAR@popcouncil.org projsoar.org

(OPopulation Council, January 2021 